\title{
Systematic cloning and analysis of autophagy-related genes from the silkworm Bombyx mori
}

\author{
Xuan Zhang ${ }^{1}$, Zhan-Ying $\mathrm{Hu}^{2}$, Wei-Fang Li ${ }^{1}$, Qing-Rong $\mathrm{Li}^{2}$, Xiao-Juan Deng ${ }^{2}$, \\ Wan-Ying Yang ${ }^{2}$, Yang $\mathrm{CaO}^{2}$ and Cong-Zhao Zhou*1
}

Address: ${ }^{1}$ Hefei National Laboratory for Physical Sciences at Microscale and School of Life Sciences, University of Science and Technology of China, Hefei Anhui 230027, PR China and 2Department of Sericulture science, College of Animal Science, South China of Agricultural University, Guangzhou 510642, PR China

Email: Xuan Zhang - xuanz@mail.ustc.edu.cn; Zhan-Ying Hu - shiyanxia@stu.scau.edu.cn; Wei-Fang Li - liwf@ustc.edu.cn; QingRong Li - liqr7702@yahoo.com.cn; Xiao-Juan Deng - dengxj@scau.edu.cn; Wan-Ying Yang - emilyywy@scau.edu.cn;

Yang Cao - caoyang@scau.edu.cn; Cong-Zhao Zhou* - zcz@ustc.edu.cn

* Corresponding author

Published: 27 May 2009

BMC Molecular Biology 2009, 10:50 doi:10.1186/1471-2199-10-50
Received: 13 December 2008

Accepted: 27 May 2009

This article is available from: http://www.biomedcentral.com//47/-2/99/10/50

(C) 2009 Zhang et al; licensee BioMed Central Ltd.

This is an Open Access article distributed under the terms of the Creative Commons Attribution License (http://creativecommons.org/licenses/by/2.0), which permits unrestricted use, distribution, and reproduction in any medium, provided the original work is properly cited.

\begin{abstract}
Background: Through the whole life of eukaryotes, autophagy plays an important role in various biological events including development, differentiation and determination of lifespan. A full set of genes and their encoded proteins of this evolutionarily conserved pathway have been identified in many eukaryotic organisms from yeast to mammals. However, this pathway in the insect model organism, the silkworm Bombyx mori, remains poorly investigated.

Results: Based on the autophagy pathway in several model organisms and a series of bioinformatic analyses, we have found more than 20 autophagy-related genes from the current database of the silkworm Bombyx mori. These genes could be further classified into the signal transduction pathway and two ubiquitin-like pathways. Using the mRNA extracted from the silkgland, we cloned the full length cDNA fragments of some key genes via reverse transcription PCR and 3' rapid amplification of CDNA ends (RACE). In addition, we found that the transcription levels of two indicator genes BmATG8 and BmATGI2 in the silkgland tend to be increased from $\left.\right|^{\text {st }}$ to $8^{\text {th }}$ day of the fifth instar larvae.

Conclusion: Bioinformatics in combination with RT-PCR enable us to remodel a preliminary pathway of autophagy in the silkworm. Amplification and cloning of most autophagy-related genes from the silkgland indicated autophagy is indeed an activated process. Furthermore, the timecourse transcriptional profiles of BmATG8 and BmATG $/ 2$ revealed that both genes are up-regulated along the maturation of the silkgland during the fifth instar. These findings suggest that the autophagy should play an important role in Bombyx mori silkgland.
\end{abstract}

\section{Background}

The programmed cell death (PCD) is a genetically regulated program of cell elimination, which is evolutionarily conserved in eukaryotes and plays a very important role in several physiological processes. PCD consists of two major types, apoptosis (type I) and autophagic cell death (type II) [1]. For a long time, autophagy has been described as a form of type II programmed cell death char- 
acterized by lysosomal activation and formation of autophagosomes. It is ubiquitous among eukaryotes functioning as a lysosome degradation pathway for recycling cytoplasmic materials especially long-lived proteins [2-4].

The formation of autophagosomes depends on the two ubiquitin-like conjugation systems, Atg8-PE (phosphatidylethanolamine) and Atg12-Atg5-Atg16 systems [5]. To trigger the former system, the $\mathrm{C}$-terminal glycine residue of newly synthesized Atg8 has to be exposed by a cysteinetype endopeptidase Atg4. Subsequently, after being activated by an E1-like protein Atg7, Atg8 is conjugated to PE by a specific E2-like protein Atg3 [6]. In the latter system, Atg12 is activated by Atg7 and then transferred to an E2like enzyme Atg10, via which conjugated to Atg5. Finally, the Atg12-Atg5 conjugate interacts with Atg16 to form a complex, which is localized to a membrane system to facilitate the maturation of autophagosomes [7-12].

There are several major signal transduction pathways and complexes involved in the induction of autophagy. One of these pathways is mediated by a serine/threonine kinase TOR (target of rapamycin), which takes part in most regulatory pathways in response to the changes in nutrient conditions and energy metabolism. TOR exerts an inhibitory effect on autophagy through various downstream effectors including Tap42 and P70s6 kinase (P70s6K) to regulate the transcription and/or translation of other related genes [13-18]. In addition to TOR, members in PtdIns 3-kinase class I (PI3K-I) and III (PI3K-III) also participate the regulation of autophagy. PI3K-I members phosphorylate PtdIns $(4,5) \mathrm{P} 2$ to produce PtdIns $(3,4,5) \mathrm{P} 3$, which binds to protein kinase B (Akt/ $\mathrm{Pkb}$ ) and its activator Pdk1 (phosphoinositide dependent kinase 1) [19-21]. The activation of PI3K-I/Akt pathway inhibits the GTPase-activating activity of Tsc2, leading to the relief of the inhibitory effect of Rheb-GDP on TOR/ P70s6K signalling [22-26]. Mammalian hormones glucagon and insulin inhibit TOR by down-regulating PI3K-I, and so does ecdysone in D. melanogaster. The PI3K-I/Akt pathway is thought to down regulate the autophagy level, while PI3K-III, together with its membrane adaptor and autophagy protein Atg6 (Beclin1 in mammals), functions as an activator of autophagy and plays a crucial role in the early steps of autophagosome formation [27-32].

Recently, an explosion of studies on autophagy and cell survival indicates that autophagy may play an important role in the life cycle of eukaryotic organism. Autophagy may help cells to survive in death mutants, to crosstalk with the regulation of cell proliferation, to remove toxic cytoplasmic constituents, to reduce neurotoxicity of polyglutamine expansion proteins in some neurodegenerative diseases and also to be required for necrotic cell death. [33-38]. The metamorphic development from larvae to pupa is accompanying with the degeneration of specific larval tissues, such as the salivary glands of Drosophila melanogaster [39-41], intersegmental muscles [42], prothoracic glands [43] and silkglands of $B$. mori [44].

The silkgland is the largest tissue in the fifth and last instar of the silkworm B. mori. It consists of three parts: anterior (ASG), middle (MSG) and posterior silkgland (PSG)[45]. During the prepupal period, silkglands are degenerated via PCD pathway triggered by the steroid hormone ecdysone $[44,46]$. Both apoptotic and autophagic morphologies have been observed in the ASG during the larvalpupal metamorphosis, but the connections between them are still unclear [47-49]. The pupal differentiation of silkgland starts on the first day of the fifth instar and cell death in ASG is initiated on the third day [44]. Besides the PCD phenomenon found in ASG, we have also observed autophagic vacuolar formation in MSG during the prepupal period (unpublished data, Cao et al.). These findings indicate that autophagy may play a very important part in the differentiation and degeneration of silkgland, but the molecular mechanism remains unclear. Thus we performed a genome-wide search of autophagy-related genes in $B$. mori, aiming at remodelling the preliminary autophagy pathway for further systematic investigations.

\section{Results \\ Identification of autophagy-related genes in B. mori}

The autophagy-related genes from databases of $S$. cerevisiae, D. melanogaster, A. mellifera and H. sapiens genome were collected as queries. Using the blast website SilkwormBLAST to search against the Silkworm Genome Database (SilkDB), more than 20 autophagy-related genes could be identified as the top hits. However, even after a series of more sensitive profile-based searches, other members remain absent probably due to the low level of sequence homology. All hits of an E-value $<1 \mathrm{e}-10$ and alignment of $>25 \%$ were collected and listed in Table 1 . Most of these genes are involved in the two ubiquitinlike conjugation systems and the upstream signal transduction pathway. Besides, we also collected some important proteins taking part in the regulation of autophagy, including the protein serine/threonine kinase Atg1 [50], integral membrane protein Atg9 [51], phosphoinositide binding protein Atg18 [52] and the heat shock cognate protein HSC73 [53] (Table 1).

\section{Remodelling the autophagy pathway}

Autophagy-related genes were first identified in yeast, and their homologs have recently been found and functionally characterized in higher eukaryotes. Evolutionary analyses indicated that autophagy is a highly conserved process among eukaryotes, such as yeast, plant, insect and mammal [54]. Based on the current knowledge, we remodel a preliminary autophagy pathway in B. mori (Fig- 
Table I: Autophagy-related proteins identified from the SilkDB.

\begin{tabular}{|c|c|c|}
\hline Autophagy proteins & Bombyx mori genes & Biochemical properties \\
\hline \multicolumn{3}{|c|}{ Ubiquitin-like conjugation pathway } \\
\hline Atg3 & BGIBMGA003767-TA & E2-like enzyme conjugates Atg8 to phosphatidylethanolamine (PE) \\
\hline Atg 4 & BGIBMGA004926-TA & Cysteine protease, cleaves C-terminal extension or PE from Atg8 \\
\hline Atg5 & BGIBMGA007878-TA & Conjugates to Atg 12 through internal lysine \\
\hline Atg7 & BGIBMGA00I 467-TA & EI-like enzyme activates Atg8 and Atg 12 \\
\hline Atg8 & BGIVMGA0I I 783-PA & Conjugates with Atg7, Atg3 \\
\hline Atg 12 & BGIBMGA003954-TA & Conjugates to Atg5 by EI enzyme Atg7 \\
\hline Atg 16 & BGIBMGA006504-TA & Protein interacts with Atg $12-A \operatorname{tg} 5$ \\
\hline \multicolumn{3}{|c|}{ Signal transduction pathway } \\
\hline PI3K-I & BGIBMGA0I056I-TA & Relieves the inhibitory effects on TOR through PI3K/Akt pathway \\
\hline Pdkl & BGIBMGAOI I 755-TA & The activator of $A k t / P k b$ \\
\hline $\mathrm{Akt} / \mathrm{Pkb}$ & BGIBMGAOI 4 I32-TA & Protein kinase B, be activated by PdkI \\
\hline Tscl & BGIBMGA005845-TA & Forms a complex with Tsc2 \\
\hline Tsc2 & BGIBMGA005686-TA & Tuberous sclerosis complex 2 , induce autophagy by inhibiting TOR \\
\hline Rheb & BGIBMGA006257-TA & Be negatively regulated by TSC2 and positively regulates TOR \\
\hline AMPK & BGIBMGAOI3/39-TA & AMP activated protein kinase, stimulates autophagy by inhibiting TOR signal pathway \\
\hline TOR & BGIBMGA008952-TA & Rapamycin target and a kinase involved in repression of autophagy \\
\hline Tap42 & BGIBMGA0I35/7-TA & A target of the TOR kinases \\
\hline $\mathrm{Pp} 2 \mathrm{~A}$ & BGIBMGA0I083I-TA & Protein phosphatase $2 \mathrm{~A}$, functions downstream of TOR \\
\hline P70s6K & BGIBMGAOI I 088-TA & A downstream effector of TOR, positively regulates autophagy \\
\hline PI3K- III & BGIBMGA007I58-TA & Contributes to the formation of autophagic vacuoles \\
\hline \multicolumn{3}{|r|}{ esmp } \\
\hline $\mathrm{Hsc73}$ & BGIBMGA00238I-TA & Involved in chaperone-mediated autophagy \\
\hline Atgl & BGIBMGA0I /986-TA & Ser/Thr kinase, required for autophagy \\
\hline Atg 18 & BGIBMGA007298-TA & Required for recycling of Atg9 \\
\hline Atg9 & BGIBMGA0I2307-PA & Transmembrane protein, formation of CVT and autophagic vesicles \\
\hline
\end{tabular}

aHomologues were identified by a BLAST search against the Silkworm Genome Database (SilkDB).

bThe alignment parameters were set as E-value of $<\mathrm{I}$ e- 10 and alignment of $>25 \%$.

ure 1). In the upstream signal transduction pathway, autophagic responses are developed by a series of kinases and phosphatases. TOR and PI3K are the major regulators in this signal transduction process (Figure 1A). In the Atg8-PE ubiquitin-like conjugation system, the last residue Asn117 of BmAtg8 is proposed to be cleaved by BmAtg4 for exposure of a C-terminal glycine residue. Then the modified BmAtg8 is activated by the E1-like enzyme BmAtg7 and finally transferred to PE by E2-like enzyme BmAtg3. In the Atg12-Atg5-Atg16 system, BmAtg12 should be activated by BmAtg7 to form a complex with BmAtg5. Subsequently, the BmAtg12-BmAtg5 conjugate interacts with BmAtg16, a protein containing a coiled-coil domain that mediates self-multimerization and the formation of an assembly of $\sim 350-\mathrm{kDa}$ (Figure 1B). All members in Atg12-Atg5-Atg16 conjugation pathway have been identified from the SilkDB except for Atg10 (colored in grey) (see the Discussion for more details).

\section{Most autophagy-related genes are actively transcribed in the silkgland}

From the $6^{\text {th }}$ day through the fifth instar, both apoptotic and autophagic morphologies in the ASG [44] and MSG (unpublished data, Cao et al.) have been observed. We extracted total RNA from the silkglands of fifth instar larvae on the $3^{\text {rd }}$ day to validate these in silico identified genes by reverse-transcription PCR. Some representative PCR products were shown in Figure 2. BmATG3, BmATG4, BmATG8 and BmATG12 are genes involved in ubiquitinlike conjugation systems; BmATG6, BmATG1 and $B m A \operatorname{tg} 18$ are required for formation of autophagosomes; Homologs of P70S6K, PKB and Rheb are members of the upstream signal transduction pathways;HSC73 mediates the chaperone-mediated autophagy.

Furthermore, full-length cDNAs of BmATG3, BmATG4, BmATG6, BmATG8 and BmATG12 have been sequenced and deposited in GenBank under the accession numbers of FJ416327, FJ416326, FJ416328, FJ416330 and Fu416329, respectively. All proteins encoded by these genes are homogeneous to their orthologs in other eukaryotes (for the multialignments of Atg4, Atg6 and Atg5, see Additional file 1), indicating high conservation of the ubiquitin-like pathways in B. mori.

In the SilkDB, we found only a $5^{\prime}$ fragment of putative BmATG5. To obtain its full-length cDNA, we used 3' RACE PCR method to amplify the unknown $3^{\prime}$ flanking 


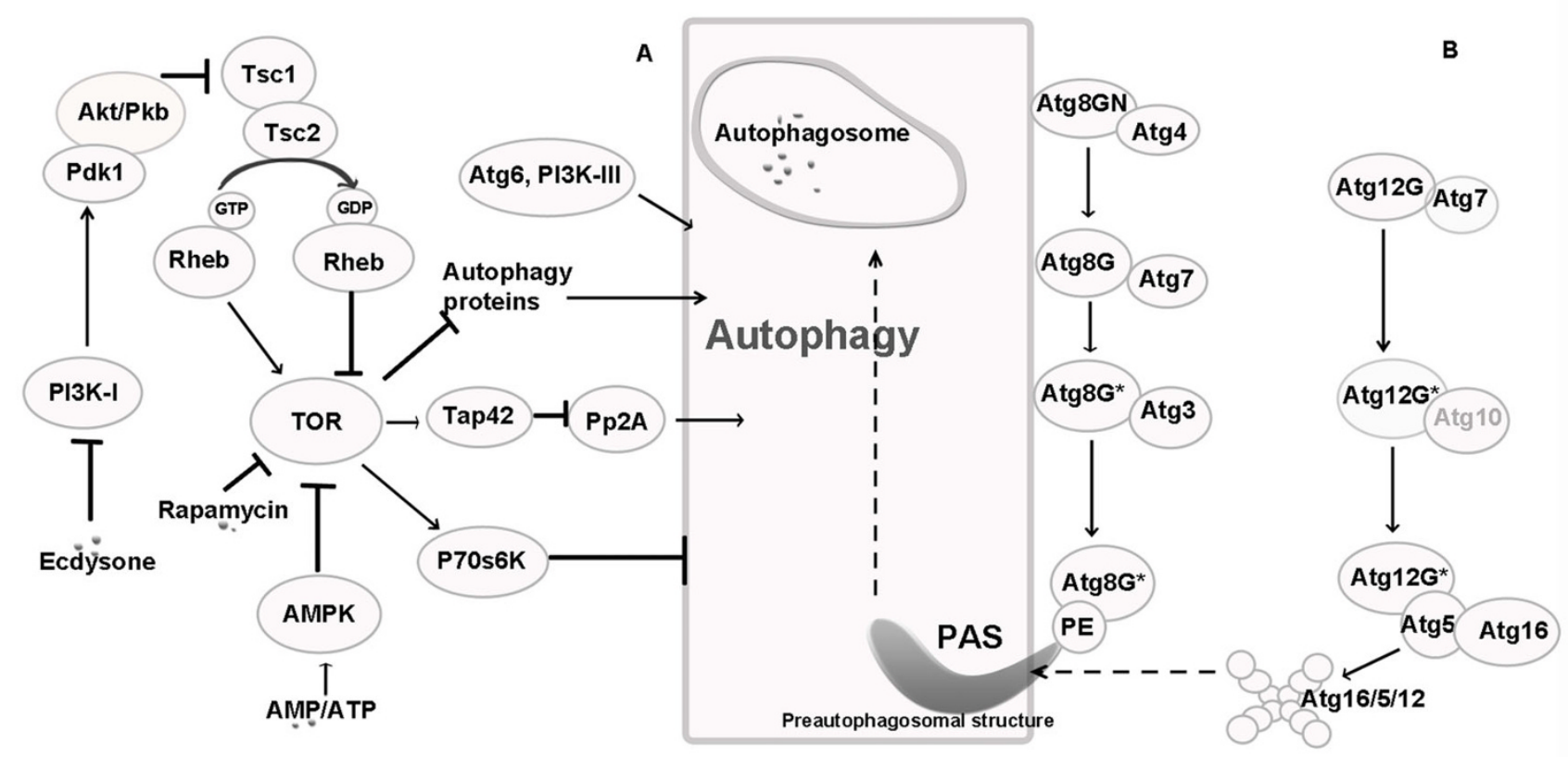

\section{Figure I}

Autophagy pathway in Bombyx mori. A) Signal transduction pathway. B) Two ubiquitin-like protein conjugation systems. The absent Atg 10 is colored in grey.

sequences. First-strand cDNA reverse-transcribed with an oligo(dT)-adaptor primer from total RNA was used as the template for 3' RACE PCR with gene specific primer GSP1 and out primer. The 3 ' RACE PCR product of BmATG5 was inserted into pGM-T vector for sequencing, and then this 3' segment was spliced with sequence of BGIBMGA007878-TA (SilkDB) to obtain the full-length cDNA sequence of BmATG5. The complete coding sequence of BmATG5 (amplified with full-length primers) has been deposited in GenBank under the accession number of FJ418152. Compared with the orthologs in other eukaryotes, BmAtg5 has the two highly conserved Atg16 binding domains (ubiquitin-like folds) and one Atg12 conjugation site (residue Lys149) (see Additional file 1).

Successful amplification of the coding sequences of some key autophagy-related genes listed in Table 1 indicated

\section{BmATG3 BmATG4 BmATG6 BmATG8 BmATG12 BmATG18 Rheb P70S6K HSC73 PKB BmATG1}

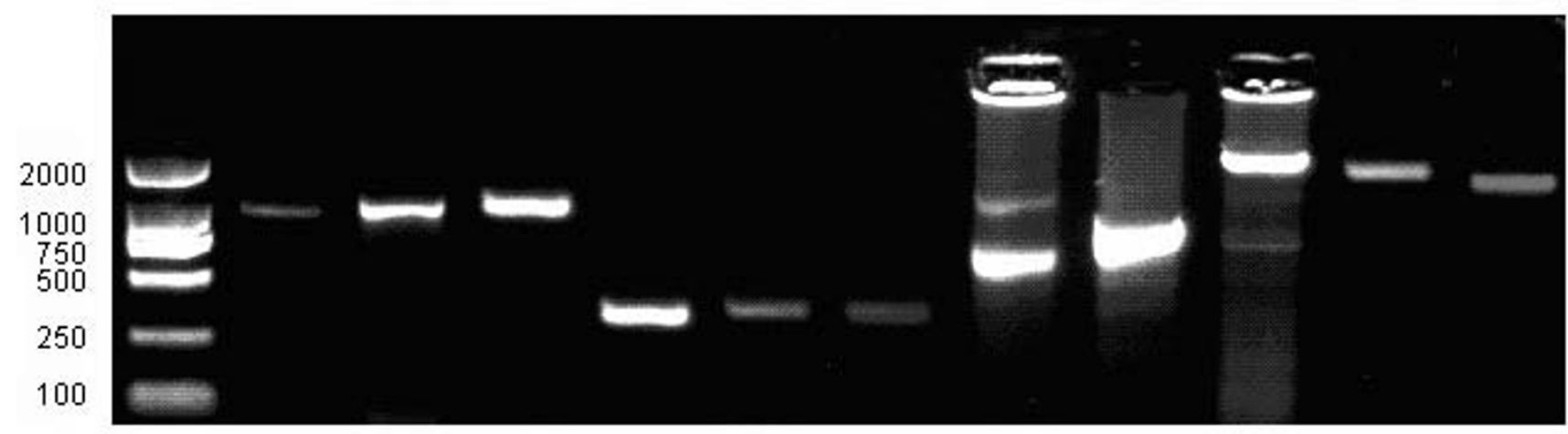

Figure 2

Some autophagy-related genes in Bombyx mori amplified by PCR with specific primers. 


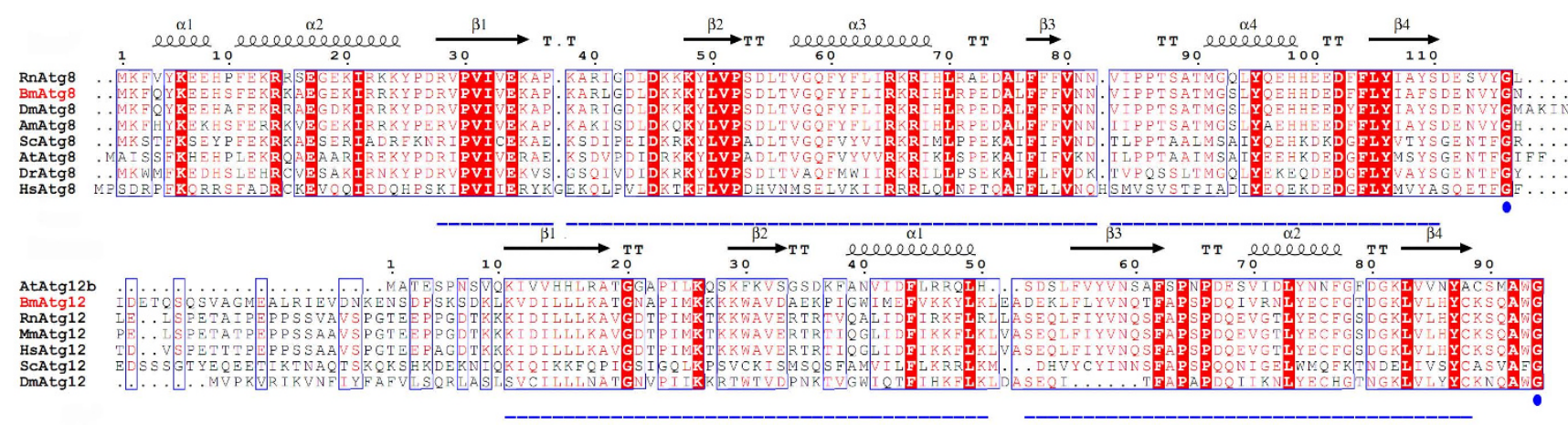

\section{Figure 3}

Multiple alignments of A) Atg8 and B) Atg 12 homologs. The ubiquitin-like fold and the C-terminal glycine are marked in blue. All sequences were obtained from NCBI, SGD and SilkDB database, alignments were performed using the programs MultAlin [56] and ESPript [57]. Species abbreviations are Bm for B. mori, Dm for D. melanogaster, Sc for S. cerevisiae, Rn for R. norvegicus, $\mathrm{Mm}$ for $\mathrm{M}$. musculus, $\mathrm{Hs}$ for $\mathrm{H}$. sapiens, At for A. thaliana, Am for A. mellifera and $\operatorname{Dr}$ for $D$. rerio.
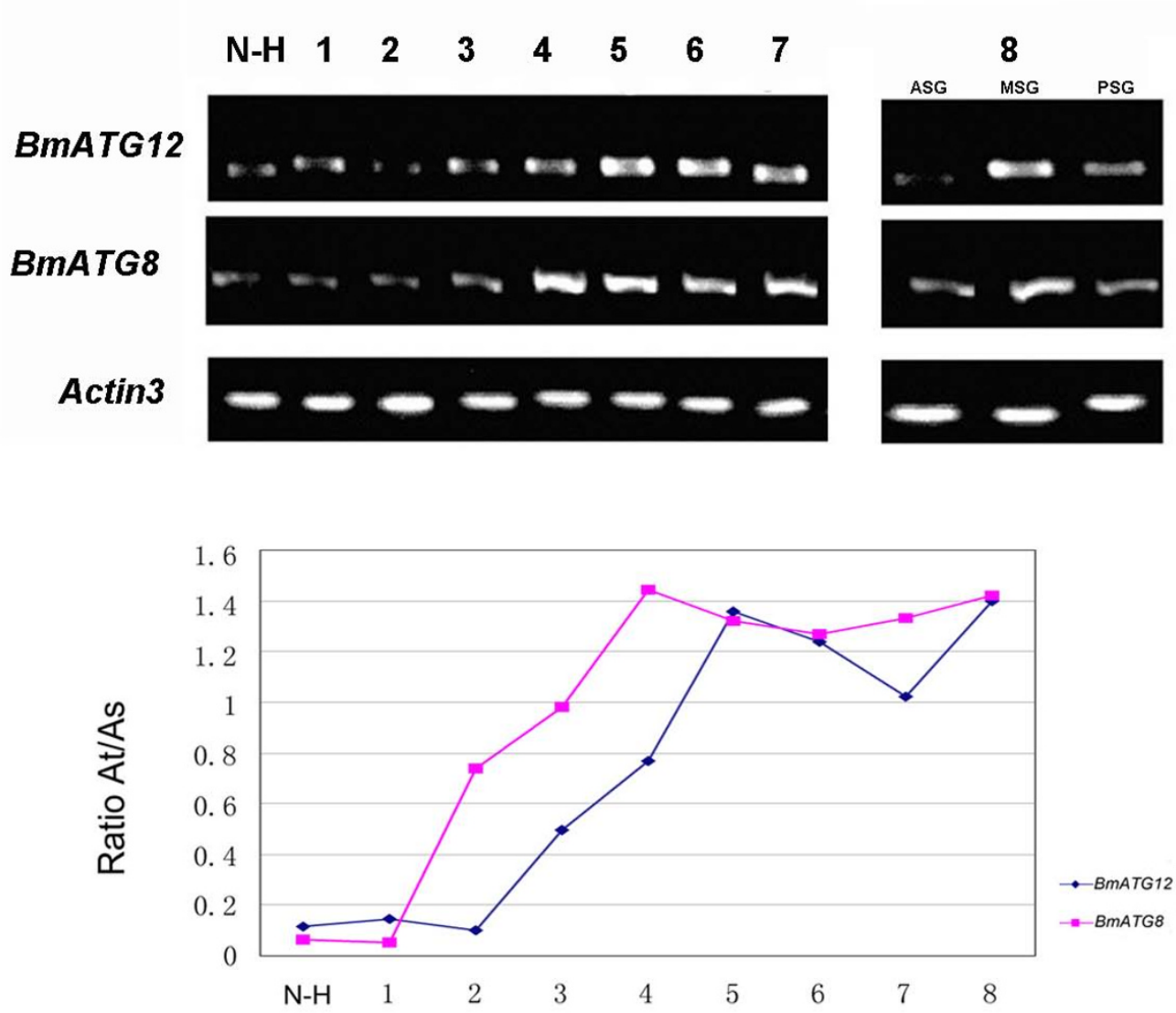

Figure 4

Semi-quantitative PCR analyses of BmATG8 and BmATGI2. A) Lane I, Total RNA from newly hatched larva (N-H); Lane 2-8, Total RNA from silkgland of $\left.\right|^{\text {st }}$ day to $7^{\text {th }}$ day fifth instar larvae ( I-7); Lane 9-I I, Total RNA from ASG(anterior silkgland), MSG(middle silkgland) and PSG(posterior silkgland) of the $8^{\text {th }}$ day fifth instar larvae. B) Quantification of the bands was performed by GIS ID Software (Tanon, Shanghai, China) and the ratio of amplified target (At) to standard (As) was calculated for all samples. 
Table 2: Databases used for bioinformatic analysis and primers for $P C R$

\begin{tabular}{ll}
\hline Databases & \\
\hline SGD & $\underline{\text { http://www.yeastgenome.org/ }}$ \\
SilkBase & $\underline{\text { http://papilio.ab.a.u-tokyo.ac.jp/silkbase }}$ \\
SilkDB & $\underline{\text { http://silkworm.genomics.org.cn/ }}$ \\
GenBank & $\underline{\text { http://www.ncbi.nlm.nih.gov/Genbank/index.html }}$ \\
FlyBase & $\underline{\text { http://flybase.bio.indiana.edu/ }}$ \\
SilkwormBLAST & $\underline{\text { http://silkworm.swu.edu.cn/silksoft/blast2-simple.html }}$ \\
\hline Primers & \\
\hline BmATG8-FOR & \\
BmATG8-REV & CATGCCATGGATGAAATTCCAATACAA \\
BmATGI2-FOR, & GCGTCGACTTAATTTCCATAGACAT \\
BmATGI2-REV & CATGCCATGGATGAGTGATGAGAAA \\
Actin3-FOR & GTGAATTCTCAGCCCCAAGCTTG \\
Actin3-REV & AGAGGTTCCGTTGTCCCG, \\
\hline
\end{tabular}

aSGD, SilkBase, SilkDB, FlyBase and SilkwormBLAST were used for bioinformatic analysis, cDNA sequences were submitted to GenBank.

bThe primers were used for semi-quantitative PCR analysis of BmATG8 and BmATGI2.

that they are indeed actively transcribed in the silkgland. Comparison to the orthologs further validated the high level of conservation of the two ubiquitin-like conjugation systems in the silkworm B. mori.

\section{Both BmATG8 and BmATGI 2 are up-regulated along the maturation of silkgland as revealed by semi-quantitative PCR}

In attempt to monitor the expression pattern of some indicator genes in the silkgland at different stages of the fifth instar, we performed a time-course transcriptional profiling of BmATG8 and BmATG12. In mammalian cells, the protein levels of Atg5-Atg12 and/or LC3-II (Atg8 homologue) have been used as indicators for the autophagic activity [55]. Multiple alignments [56,57] indicated that both Atg8 and Atg12 homologs have an ubiquitin-like fold and a highly conserved glycine residue at the C-terminal region (Figure 3), which is essential for their conjugation to Atg3 and Atg5, respectively. Therefore, we used the transcription levels of BmATG12 and BmATG8 to indicate the autophagic activity in the silkgland of B. mori.

Total RNA from the silkgland on each day in the fifth instar larvae was prepared for reverse-transcription. Double-stranded cDNA samples of the same quantity were used as templates for semi-quantitative PCR with primers for BmATG8 and BmATG12, respectively. The quantification of the bands was performed by GIS 1D Software (Tanon, Shanghai, China) and ratio of amplified target BmATG8/BmATG12 to standard Actin3 was calculated. As shown in Figure 4, the transcriptional levels of both BmATG8 and BmATG12 exhibit an overall tendency of increase from $1^{\text {st }}$ to $8^{\text {th }}$ day, and reach a plateau on the $4^{\text {th }}$ day (BmATG8) and the $5^{\text {th }}$ day (BmATG12) of fifth instar larvae, respectively. These results suggested a significant up-regulation of autophagic level in the silkgland of fifth instar larvae, which is in agreement with the previous histochemical investigations [47]. Thus we suggested that autophagy should take a crucial part in the differentiation and degeneration of the silkgland in prepupal of B. mori.

\section{Discussion}

Along the metamorphic development from larvae to pupa, some larval organs of $B$. mori such as the larval midgut, intersegmental muscles and silkglands are degenerated and new imaginal structures are developed. The histolysis of these tissues was proposed to be achieved via programmed cell death and the degradation process in the ASG cells display both autophagic and apoptotic characteristics $[44,46,58]$. However, the $B$. mori genes or proteins required for autophagy have not been well characterized as those identified from the yeast or human [59].

In the present work, we performed a comprehensive bioinformatic analysis and found 24 autophagy-related proteins as the top hits from the SilkDB (Table 1). All homologs involved in the two ubiquitin-like pathways have been found in $B$. mori except for Atg10 which has not been identified from other two model insects, neither fruitfly nor honeybee. As well known, the E2-like enzyme Atg10 plays a very important part in transferring Atg12 to Atg5. It remains a puzzle that how the Atg12-Atg5-Atg16 conjugation system works without a functional Atg10. After performing a multiple alignment of the primary sequences of Atg10 against those of E2-like enzyme Atg3 from different species, we found that Atg3 proteins of some insects have low homology to mammalian Atg10. 
As shown in Additional file 2, both Atg3 and Atg10 have a highly conserved C-terminal catalytic cysteine (marked in blue) which is essential for the conjugation [60]. BmAtg3 might be able to recognize both BmAtg8 and BmAtg12 which share an ubiquitin-like fold and a highly conserved glycine residue at the $\mathrm{C}$-terminal region (Figure 3 ). Thus we hypothesize that the function of Atg10 could be compensated by Atg3 in B. mori and other lepidoptera insects. Further in vivo experiments, especially identification of the E2-like enzyme of Atg12, are necessary to verify this hypothesis.

Based on these 24 genes and their high degree of conservation to the orthologs identified and functionally characterized in higher eukaryotes [4,61-63], a preliminary autophagy pathway in B. mori was remodelled (Figure 1). In addition to these in silico analyses, RT-PCR results strongly indicated that autophagy-related genes in ubiquitin-like conjugation systems are indeed actively transcribed in the silkgland. This is in agreement with the previous report that $B$. mori silkglands are committed to undergo PCD from the $3^{\text {rd }}$ day of the fifth instar and are degenerated completely soon after pupation [44]. To verify the temporal control of this process, we performed a time-course transcriptional profiling of two indicator genes BmATG8 and BmATG12 via semi-quantitative RTPCR analysis. As expected, the transcriptional levels of both BmATG8 and BmATG12 exhibit a general tendency of increase from $1^{\text {st }}$ to $8^{\text {th }}$ day.

\section{Conclusion}

As an evolutionarily conserved and finely regulated process, autophagy plays a very important role in various biological events during the whole life of eukaryotes, including the metamorphic development of B. mori. In the present work, we identified over 20 autophagy-related genes and remodelled the autophagy pathway in $B$. mori. Using the total RNA extracted from the silkgland of the fifth instar larvae on the $3^{\text {rd }}$ day as the initial template, the coding sequences of several key genes were further cloned by reverse transcription PCR and/or 3' RACE PCR experiments, indicating that most autophagy-related genes are actively transcribed in the silkgland. Furthermore, semiquantitative PCR results indicated that both BmATG8 and $B m A T G 12$ are up-regulated in fifth instar larvae of $B$. mori. Taken together, our findings, in combination with the previous histochemical investigations, evidently suggest that autophagy executes a very important role in the differentiation and degeneration of silkgland during the metamorphosis from larva to pupa.

\section{Methods}

\section{Bioinformatic analyses}

All autophagy-related genes in yeast and homologs in $D$. melanogaster, A. mellifera, $H$. sapiens, $R$. norvegicus, M. mus- culus, A. thaliana, A. mellifera, and D. rerio were collected as queries for BLAST search against the silkworm database SilkDB, and then the top hits were selected for annotation. The databases used for sequence analyses include SGD, SilkBase, SilkDB, GenBank, FlyBase and SilkwormBLAST (Table 2). Full-length sequences of autophagyrelated genes found from the SilkDB were listed in Table 1. The parameters for BLAST were set as E-value of $<1 \mathrm{e}-10$ and alignment of $>25 \%$.

\section{RNA extraction, CDNA preparation and PCR validation of some key genes}

Total RNA extracted (Trizol, Invitrogen) from the silkglands of the fifth instar larvae at the $3^{\text {rd }}$ day was reversetranscribed with Super Script III (Invitrogen). Primers were designed according to the sequences downloaded from SilkDB Database. The cDNA prepared from total RNA was used as template for PCR amplification in a volume of $25 \mu \mathrm{l}$ with corresponding primers. The PCR products were separated on $1.5 \%$ agarose gel stained with GelRed.

The 3' end CDNA sequence of BmATG5 was obtained by using 3' RACE PCR with total RNA from mesenteron of $B$. mori in fifth instar. First strand cDNA was synthesized using an Oligo(dT)-Adaptor primer provided by Takara 3' RACE kit at $42^{\circ} \mathrm{C}$ for $60 \mathrm{~min}$ in a reaction volume of 10 $\mu \mathrm{l}$. Then the cDNA was amplified using the 3' RACE Outer Primer (TACCGTCGTTCCACTAGTGATTT) and BmATG5 specific primer GSP1 (GGACTTTGACAGTACACTTC) as following procedure: $5 \mathrm{~min}$ at $94^{\circ} \mathrm{C}, 35$ cycles of $30 \mathrm{sec}$ at $94^{\circ} \mathrm{C} 30 \mathrm{sec}$ at $55^{\circ} \mathrm{C}, 66 \mathrm{sec}$ at $72^{\circ} \mathrm{C}$, and then $10 \mathrm{~min}$ at $72^{\circ} \mathrm{C}$. The PCR product was purified and cloned into pGM-T sequencing vector. According to the sequencing result, we spliced out the full-length sequence of BmATG5 and obtained it using PCR reaction with full-length primers.

\section{Semi-quantitative PCR analysis of BmATG8 and BmATG I 2}

Total RNA from silkgland of each day in fifth instar larvae was reverse-transcribed to cDNA. The cDNAs of the same quantity was used as template for semi-quantitative PCR amplification in a volume of $20 \mu \mathrm{l}$ with BmATG8, BmATG12 and Actin3 primers (Table 2). Each PCR reaction was performed by preheating the samples at $94^{\circ} \mathrm{C}$ for $5 \mathrm{~min}$ followed by 20 cycles of $94^{\circ} \mathrm{C}$ for $30 \mathrm{sec}, 55^{\circ} \mathrm{C}$ for $30 \mathrm{sec}$ and $72^{\circ} \mathrm{C}$ for $40 \mathrm{sec}$ followed by an extra extension at $72^{\circ} \mathrm{C}$ for $10 \mathrm{~min}$, the PCR products were separated on $1.5 \%$ agarose gel. Quantification of the bands was performed by GIS 1D Software (Tanon, Shanghai, China) and band intensities were expressed as relative absorbance units Volume $(\mu \mathrm{g})$. The ratio of amplified BmATG 8 and BmATG12 respectively to Actin3 was calculated for all samples. 


\section{Authors' contributions}

$\mathrm{XZ}$ performed the bioinformatic analyses, RNA extraction and RT-PCR, semi-quantitative PCR. YZH performed 3' RACE PCR of BmATG5 and obtained the full length cDNA sequence of some genes. CZZ and WFL coordinated the project and provided financial support. XZ and CZZ designed the experiments and wrote the paper. All authors have read and approved the final manuscript.

\section{Additional material}

\section{Additional file 1 \\ Multiple alignments of A) Atg4, B) Atg6, and C) Atg5 homologs. The catalytic residues of Atg4 are marked in blue. The conserved conjugation site to Atg12 in Atg5 is marked in blue, the ubiquitin-like folds UblA and UblB (marked with blue dashed line) are essential for the binding of Atg 5 to Atg16. All sequences were obtained from NCBI, SGD and SilkDB database, alignments were performed using the programs MultAlin [56] and ESPript [57]. Species abbreviations are $\mathrm{Bm}$ for $\mathrm{B}$. mori, $\mathrm{Dm}$ for $\mathrm{D}$. melanogaster, Sc for $\mathrm{S}$. cerevisiae, Rn for $\mathrm{R}$. norvegicus, $\mathrm{Mm}$ for $\mathrm{M}$. musculus, Hs for H. sapiens, At for A. thaliana, Am for A. mellifera and $\mathrm{Dr}$ for $\mathrm{D}$. rerio. \\ Click here for file \\ [http://www.biomedcentral.com/content/supplementary/1471- 2199-10-50-S1.tiff] \\ Additional file 2 \\ Multiple alignment of the primary sequences of Atg10 against Atg3 from different species. The highly conserved catalytic cysteines of Atg 3 homologs are marked in blue; The HR domain of Atg 3 and the catalytic cysteine are marked with blue dashed line and dot, respectively. Species abbreviations are $\mathrm{Bm}$ for $\mathrm{B}$. mori, Am for A. mellifera; Dm for D. mel- anogaster, Ag for A. gambiae, Mm for M. musculus, Hs for H. sapi- ens, Rn for R. norvegicus, Sc for S. cerevisiae and At for A. thaliana. Click here for file \\ [http://www.biomedcentral.com/content/supplementary/1471- 2199-10-50-S2.tiff]}

\section{Acknowledgements}

We thank Prof. Qingyou Xia at the Key Sericultural Laboratory of Agriculture Ministry, Southwest University of China for providing some RNA samples. This work is sponsored by the Chinese National Natural Science Foundation (Grant 90608027), the Hi-Tech Research and Development Program of China (Grant 2006AA I0AII 19) and the 973 project (Grant 2005CB I 2 1002) from the Ministry of Science and Technology of China, and the Knowledge Innovation Program from Chinese Academy of Science.

\section{References}

I. Abraham MC, Shaham S: Death without caspases, caspases without death. Trends in cell biology 2004, 14:184-193.

2. Mortimore GE, Poso AR: Intracellular protein catabolism and its control during nutrient deprivation and supply. Annual review of nutrition 1987, 7:539-564.

3. Klionsky DJ, Emr SD: Autophagy as a regulated pathway of cellular degradation. Science 2000, 290(5497): |7|7-|72|

4. Yorimitsu T, Klionsky DJ: Autophagy: molecular machinery for self-eating. Cell Death Differ 2005, I 2: I542-I552.

5. Tanida I, Ueno T, Kominami E: LC3 conjugation system in mammalian autophagy. The international journal of biochemistry \& cell biology 2004, 36(1 2):2503-25 I8.
6. Kirisako $\mathrm{T}$, Ichimura $\mathrm{Y}$, Okada $\mathrm{H}$, Kabeya $\mathrm{Y}$, Mizushima $\mathrm{N}$, Yoshimori T, Ohsumi M, Takao T, Noda T, Ohsumi Y: The reversible modification regulates the membrane-binding state of Apg8/Aut7 essential for autophagy and the cytoplasm to vacuole targeting pathway. The Journal of cell biology 2000, I 5 I (2):263-276.

7. Komatsu M, Tanida I, Ueno T, Ohsumi M, Ohsumi Y, Kominami E: The C-terminal region of an Apg7p/Cvt2p is required for homodimerization and is essential for its EI activity and EIE2 complex formation. Journal of Biological Chemistry 200I, 276(I3):9846-9854.

8. Mizushima N, Yamamoto A, Hatano M, Kobayashi Y, Kabeya Y, Suzuki $\mathrm{K}$, Tokuhisa T, Ohsumi Y, Yoshimori T: Dissection of autophagosome formation using Apg5-deficient mouse embryonic stem cells. Journal of Cell Biology 200 I, I 52(4):657-667.

9. Nemoto T, Tanida I, Tanida-Miyake E, Minematsu-lkeguchi N, Yokota M, Ohsumi M, Ueno T, Kominami E: The mouse APGIO homologue, an E2-like enzyme for Apg I 2p conjugation, facilitates MAP-LC3 modification. Journal of Biological Chemistry 2003, 278(4I):39517-39526.

10. Tanida I, Nishitani T, Nemoto T, Ueno T, Kominami E: Mammalian Apg I 2p, but not the Apg I2p center dot Apg5p conjugate, facilitates LC3 processing. Biochemical and biophysical research communications 2002, 296(5): II64-II 70.

II. Tanida I, Tanida-Miyake E, Komatsu M, Ueno T, Kominami E: Human Apg3p/Aut I p homologue is an authentic E2 enzyme for multiple substrates, GATE-16, GABARAP, and MAP-LC3, and facilitates the conjugation of hApg I 2p to hApg5p. Journal of Biological Chemistry 2002, 277(16): 13739-13744.

12. Tanida I, Tanida-Miyake E, Ueno T, Kominami E: The human homolog of Saccharomyces cerevisiae Apg7p is a proteinactivating enzyme for multiple substrates including human Apg 12p, GATE-16, GABARAP, and MAP-LC3. Journal of Biological Chemistry 200I, 276(3): I70I-I 706.

13. Beck T, Hall MN: The TOR signalling pathway controls nuclear localization of nutrient-regulated transcription factors. Nature 1999, 402(6762):689-692.

14. Chan TF, Bertram PG, Ai W, Zheng XF: Regulation of APG I4 expression by the GATA-type transcription factor GIn3p. The Journal of biological chemistry 200I, 276(9):6463-6467.

15. Fingar DC, Salama S, Tsou C, Harlow E, Blenis J: Mammalian cell size is controlled by mTOR and its downstream targets S6K I and 4EBP I/elF4E. Genes \& development 2002, I 6( I 2): I 472-I 487.

16. Huang WP, Scott SV, Kim J, Klionsky DJ: The itinerary of a vesicle component, Aut7p/Cvt5p, terminates in the yeast vacuole via the autophagy/Cvt pathways. The Journal of biological chemistry 2000, 275(8):5845-585I.

17. Jiang $Y$, Broach JR: Tor proteins and protein phosphatase $2 A$ reciprocally regulate Tap42 in controlling cell growth in yeast. The EMBO journal 1999, 18(10):2782-2792.

18. Rohde J, Heitman J, Cardenas ME: The TOR kinases link nutrient sensing to cell growth. The Journal of biological chemistry 200I, 276(13):9583-9586.

19. Katso R, Okkenhaug K, Ahmadi K, White S, Timms J, Waterfield MD: Cellular function of phosphoinositide 3-kinases: Implications for development, immunity, homeostasis, and cancer. Annual Review of Cell and Developmental Biology 200I, 17:615-675.

20. Petiot A, Ogier-Denis E, Blommaart EFC, Meijer AJ, Codogno P: Distinct classes of phosphatidylinositol 3 '-kinases are involved in signaling pathways that control macroautophagy in HT-29 cells. Journal of Biological Chemistry 2000, 275(2):992-998.

21. Vanhaesebroeck B, Leevers SJ, Panayotou G, Waterfield MD: Phosphoinositide 3-kinases: A conserved family of signal transducers. Trends in biochemical sciences 1997, 22(7):267-272.

22. Baehrecke EH: Autophagy: dual roles in life and death? Nature reviews 2005, 6(6):505-5I0.

23. Garami A, Zwartkruis FJ, Nobukuni T, Joaquin M, Roccio M, Stocker $H$, Kozma SC, Hafen E, Bos JL, Thomas G: Insulin activation of Rheb, a mediator of $\mathrm{mTOR} / \mathrm{S} 6 \mathrm{~K} / 4 \mathrm{E}-\mathrm{BP}$ signaling, is inhibited by TSCI and 2. Molecular cell 2003, I I(6): I457-I466.

24. Klionsky $D$ J: The molecular machinery of autophagy: unanswered questions. Journal of cell science 2005, I I 8(Pt I):7-18.

25. Hall DJ, Grewal SS, de la Cruz AF, Edgar BA: Rheb-TOR signaling promotes protein synthesis, but not glucose or amino acid import, in Drosophila. BMC biology 2007, 5: 10.

26. Yang Q, Inoki K, Kim E, Guan KL: TSCI/TSC2 and Rheb have different effects on TORCI and TORC2 activity. Proceedings of 
the National Academy of Sciences of the United States of America 2006, I03(18):68II-6816.

27. Yoshimori T: Autophagy: a regulated bulk degradation process inside cells. Biochemical and biophysical research communications 2004, 3 I 3(2):453-458.

28. Yue ZY, Jin SK, Yang CW, Levine AJ, Heintz N: Beclin I, an autophagy gene essential for early embryonic development, is a haploinsufficient tumor suppressor. Proceedings of the National Academy of Sciences of the United States of America 2003, 100(25): I 5077-15082.

29. Takahashi Y, Coppola D, Matsushita N, Cualing HD, Sun M, Sato Y, Liang C, Jung JU, Cheng JQ, Mule J], et al.: Bif-I interacts with Beclin I through UVRAG and regulates autophagy and tumorigenesis. Nature Cell Biology 2007, 9(I0): I | 42-II5I.

30. Liang XH, Jackson S, Seaman M, Brown K, Kempkes B, Hibshoosh H, Levine $B$ : Induction of autophagy and inhibition of tumorigenesis by beclin I. Nature 1999, 402(6762):672-676.

31. Cao Y, Klionsky DJ: Physiological functions of Atg6/Beclin I: a unique autophagy-related protein. Cell Research 2007, I 7(10):839-849.

32. Hu GW, Hacham M, Waterman SR, Panepinto J, Shin S, Liu XG, Gibbons J, Valyi-Nagy T, Obara K, Jaffe HA, et al.: PI3K signaling of autophagy is required for starvation tolerance and virulence of Cryptocloccus neoformans. Journal of Clinical Investigation 2008, I I 8(3): I I 86-II 97.

33. Xavier RJ, Huett A, Rioux JD: Autophagy as an important process in gut homeostasis and Crohn's disease pathogenesis. Gut 2008, 57(6):717-720.

34. Maiuri MC, Zalckvar E, Kimchi A, Kroemer G: Self-eating and selfkilling: crosstalk between autophagy and apoptosis. Nature Reviews Molecular Cell Biology 2007, 8(9):74I-752.

35. Levine $B$, Kroemer $G$ : Autophagy in aging, disease and death: the true identity of a cell death impostor. Cell Death Differ 2009 I6(I):I-2.

36. Kroemer G, Levine B: Autophagic cell death: the story of a misnomer. Nature Reviews Molecular Cell Biology 2008, 9( I 2): I 004-10 I0.

37. Cecconi $F$, Levine $B$ : The role of autophagy in mammalian development: Cell makeover rather than cell death. Developmental Cell 2008, I5(3):344-357.

38. Samara C, Syntichaki $P$, Tavernarakis N: Autophagy is required for necrotic cell death in Caenorhabditis elegans. Cell Death Differ 2008, I5(I): 105-III

39. Jiang CA, Lamblin AFJ, Steller H, Thummel CS: A steroid-triggered transcriptional hierarchy controls salivary gland cell death during Drosophila metamorphosis. Molecular Cell 2000, 5(3):445-455

40. Farkas R, Sutakova G: Ultrastructural changes of Drosophila larval and prepupal salivary glands cultured in vitro with ecdysone. In Vitro Cellular \& Developmental Biology-Animal 1998, 34(I0):8। 3-823.

4I. Lee CY, Baehrecke EH: Steroid regulation of autophagic programmed cell death during development. Development 200I, | 28(8): | 4433-I 455.

42. Schwartz LM: Insect Muscle as a Model for Programmed CellDeath. Journal of Neurobiology 1992, 23(9): |3 |2-1326.

43. Dai JD, Gilbert LI: Programmed cell death of the prothoracic glands of Manduca sexta during pupal-adult metamorphosis. Insect Biochemistry and Molecular Biology 1997, 27(I):69-78.

44. Terashima J, Yasuhara N, Iwami M, Sakurai S, Sakurai S: Programmed cell death triggered by insect steroid hormone, 20-hydroxyecdysone, in the anterior silk gland of the silkworm, Bombyx mori. Dev Genes Evol 2000, 21 ( (I I):545-558.

45. Akai $\mathrm{H}$ : The structure and ultrastructure of the silk gland. Cellular and Molecular Life Sciences 1983, 39:443-449.

46. Elmogy M, Iwami M, Sakurai S: Presence of membrane ecdysone receptor in the anterior silk gland of the silkworm Bombyx mori. European Journal of Biochemistry 2004, 27 I(I5):3 I7I-3I79.

47. Goncu E, Parlak O: Morphological changes and patterns of ecdysone receptor BI immunolocalization in the anterior silk gland undergoing programmed cell death in the silkworm, Bombyx mori. Acta Histochem 2009, II I (I):25-34.

48. Mpakou VE, Nezis IP, Stravopodis DJ, Margaritis LH, Papassideri IS: Different modes of programmed cell death during oogenesis of the silkmoth Bombyx mori. Autophagy 2008, 4(I):97-100.
49. Goncu E, Parlak O: Some autophagic and apoptotic features of programmed cell death in the anterior silk glands of the silkworm, Bombyx mori. Autophagy 2008, 4(8): I069-1072.

50. Reggiori F, Tucker KA, Stromhaug PE, Klionsky DJ: The Atg IAtg 13 complex regulates Atg9 and Atg 23 retrieval transport from the pre-autophagosomal structure. Developmental Cell 2004, 6(I):79-90.

51. Noda T, Kim J, Huang WP, Baba M, Tokunaga C, Ohsumi Y, Klionsky $D$ J: Apg9p/Cvt7p is an integral membrane protein required for transport vesicle formation in the Cvt and autophagy pathways. Journal of Cell Biology 2000, I 48(3):465-479.

52. Obara K, Sekito T, Niimi K, Ohsumi Y: The Atg I 8-Atg2 complex is recruited to autophagic membranes via phosphatidylinositol 3-phosphate and exerts an essential function. Journal of Biological Chemistry 2008, 283(35):23972-23980.

53. Dice JF: Chaperone-mediated autophagy. Autophagy 2007, 3(4):295-299.

54. Reggiori F, Klionsky DJ: Autophagy in the eukaryotic cell. Eukaryotic Cell 2002, I(I): I I-2I.

55. Kabeya Y, Mizushima N, Yamamoto A, Oshitani-Okamoto S, Ohsumi Y, Yoshimori T: LC3, GABARAP and GATEI6 localize to autophagosomal membrane depending on form-II formation. Journal of cell science 2004, I I 7(Pt |3):2805-28I2.

56. Corpet F: Multiple sequence alignment with hierarchical clustering. Nucleic acids research 1988, 16(22): 1088I-10890.

57. Gouet P, Robert X, Courcelle E: ESPript/ENDscript: Extracting and rendering sequence and $3 D$ information from atomic structures of proteins. Nucleic acids research 2003, 3 I(I3):3320-3323

58. Kakei M, Iwami M, Sakurai S: Death commitment in the anterior silk gland of the silkworm, Bombyx mori. Journal of Insect Physiology 2005, 5 I ( I): I7-25

59. Klionsky DJ, Cuervo AM, Seglen PO: Methods for monitoring autophagy from yeast to human. Autophagy 2007, 3(3): |8I-206.

60. Yamada Y, Suzuki NN, Hanada T, Ichimura Y, Kumeta H, Fujioka $Y$, Ohsumi $Y$, Inagaki F: The crystal structure of Atg3, an autophagy-related ubiquitin carrier protein (E2) enzyme that mediates Atg8 lipidation. The Journal of biological chemistry 2007, 282(I I):8036-8043.

6I. Huang WP, Klionsky DJ: Autophagy in yeast: A review of the molecular machinery. Cell Structure and Function 2002, 27(6):409-420.

62. Klionsky DJ: The molecular machinery of autophagy: unanswered questions. Journal of Cell Science 2005, I I 8(1):7-18.

63. Khalfan WA, Klionsky DJ: Molecular machinery required for autophagy and the cytoplasm to vacuole targeting (Cvt) pathway in S-cerevisiae. Current Opinion in Cell Biology 2002, 14(4):468-475.

Publish with Bio Med Central and every scientist can read your work free of charge

"BioMed Central will be the most significant development for disseminating the results of biomedical research in our lifetime. "

Sir Paul Nurse, Cancer Research UK

Your research papers will be:

- available free of charge to the entire biomedical community

- peer reviewed and published immediately upon acceptance

- cited in PubMed and archived on PubMed Central

- yours - you keep the copyright 